\title{
Erneuerbare Energien in Deutschland im Kontext von Umweltbewusstsein und ökologischem Wertewandel
}

Wie kam es zu der weltweit anerkannten Spitzenforschung im Bereich der erneuerbaren Energien in Deutschland? Der Artikel basiert auf einem Buchprojekt, das sich mit der Geschichte der Erforschung erneuerbarer Energien befasst. Diese Entwicklung wird anhand von Interviews mit Solarenergieforschern, die die Forschung seit den 1970er Jahren vorangetrieben haben, illustriert. Von Gerd Stadermann

$\mathbf{Z}$ ahlreiche Bücher, Filme und Radiosendungen thematisieren die Energiewende - aber es gibt kaum etwas über die Wissenschaft und die Forscher. Doch sie sind es ja, die die erneuerbaren Energiequellen nutzbar machten, wirtschaftlich, ökologisch verträglich und gesellschaftlich akzeptabel. Aus der Geschichte der Probleme mit und bei der Energiegewinnung ergibt sich für die Energiewirtschaft die Konsequenz, dass nur eine nachhaltige Energieversorgung eine Zukunft haben kann, wobei festzustellen ist, dass nur die erneuerbaren Energien den Kriterien der Nachhaltigkeit genügen. Denn die Erforschung und Entwicklung der erneuerbaren Energien erfolgt nicht als Lösung eines Schönwetterproblems einer Gesellschaft, die es sich leisten kann. Es gibt harte Fakten, die das Ziel einer nachhaltigen Energiegewinnung unausweichlich machen. Sie bilden das Fundament für die derzeitigen Entwicklungen der erneuerbaren Energien. Die Wissenschaftler/ innen und Forscher/innen, die den Entwicklungsprozess angestoßen haben, sind nicht ideologischen Strömungen gefolgt, sondern haben Verantwortung übernommen. Daraus entwickelte sich ein sozialer Auftrag, die Erneuerbaren für die Gesellschaft zu erschließen, und ein Gesellschaftsvertrag, der der wichtigste der Gegenwart sein dürfte.

Die Beschränkung auf Deutschland resultiert aus der spezifischen Geschichte Deutschlands. Diese kann zwar keineswegs vom internationalen Kontext getrennt bewertet werden, dennoch war es eine historisch einmalige Zeit, aus der heraus eine Forschung und Entwicklung entstanden ist, die Deutschland in eine Spitzenposition der erneuerbaren Technologien brachte.

\section{Motivation}

Ein Ziel dieser Untersuchung ist es, die historisch einmalige Zeit zu beschreiben, in der die Weichen in Richtung Energiewende gestellt wurden. Dabei gilt es, die Besonderheiten der ersten Etappe zur Erforschung und Entwicklung der erneuerbaren Energien in den 1970er und 1980er Jahren herauszuarbeiten und mit den damals aktiven Protagonist/innen zu verbinden.

Ein weiteres Ziel des Projektes ist es, die Frage zu beantworten, ob die Erforschung der erneuerbaren Energien als Transformationsmodell für Nachhaltigkeit auch in anderen Bereichen von Wissenschaft und Forschung von heute dienen kann.

Ein besonderes Anliegen dieses Beitrages ist es, Antwort auf die Frage zu geben, welche Rolle die Wissenschaft für eine Demokratie spielt: Warum braucht Demokratie Wissenschaft? Anhand der Forschung und Entwicklung der solaren und erneuerbaren Energien soll gezeigt werden, dass sie für eine Demokratie unabdingbar sind.

\section{Untersuchungsebenen für eine Forschungs- geschichte der erneuerbaren Energien}

Die Untersuchung der Forschungsgeschichte der erneuerbaren Energien soll erklären, warum und auf welche Weise Deutschland Weltmeister in der technologischen Entwicklung der erneuerbaren Energien geworden ist. Welche gesellschaftlichen, wirtschaftlichen, technologischen und sozialen Gründe haben zur Entwicklung der erneuerbaren Energien geführt? Wer waren die Protagonist/innen in den 1970er und 1980er Jahren? Welchen Einfluss hatten die Ölkrisen, das sich entwickelnde Umweltbewusstsein und das Einsetzen eines ökologischen Wertewandels in der Bundesrepublik seit den 1970er Jahren auf die Erforschung und Entwicklung der erneuerbaren Energien? Beginnend mit Forschungsaktivitäten an Universitäten und außeruniversitären Einrichtungen vollzog sich ein Institutionalisierungsprozess auf dem Gebiet der erneuerbaren Energien. Dabei werden die Wechselbeziehungen zwischen den energiepolitischen Rahmenbedingungen für die Gründung von Forschungseinrichtungen (Feldebene), die sich im Laufe der Entwicklung herausgebildet haben, und dem Handeln der beteiligten Akteure (Akteursebene) nachgezeichnet. Die Analysestrategie des Projekts adressiert beide Ebenen, indem sie einer- 
seits den gesellschaftlichen und politischen Kontext für die Herausbildung der Erforschung erneuerbarer Energien historisch nachzeichnet und diesen mit den subjektiven Motiven, Zielen und Leitbildern der beteiligten Akteure verknüpft.

Die Geschichte erzählt von Ereignissen und von Menschen, die durch ihr Verantwortungsbewusstsein dazu beitrugen, dass Problemlagen bei der Energieerzeugung allgemein anerkannt wurden und sich daraus ein sozialer Auftrag ergab. Mit anderen Worten: das gewachsene Umweltbewusstsein begann sich politisch zu manifestieren. Sobald aus einem sozialen Auftrag eine politische Vorgabe wird, entsteht ein gesellschaftlicher Auftrag, denn damit entwickeln sich gesetzliche beziehungsweise verbindliche Maßnahmen. Ein neuer Gesellschaftsvertrag zur Überwindung vorliegender Probleme wird geboren.

Kernstück der historischen und wissenschaftssoziologischen Untersuchung sind Interviews mit Solarenergieforscher/innen, Forschungspolitiker/innen, Beamten, Abgeordneten und Wirtschaftsvertreter/innen, die die Erforschung der erneuerbaren Energien seit den 1970er Jahren vorangetrieben haben. In ihren persönlichen Erfahrungen spiegeln sich Motivationen, Erfolge aber auch sozial- und forschungspolitische Hemmnisse, mithin die Akzeptanzgeschichte der Erforschung erneuerbarer Energien.

\section{Zeitstruktur}

Der Zeitrahmen der Untersuchung umfasst zwei Abschnitte: Der erste Abschnitt des Projekts betrachtet die Zeit vom Anfang der 1970er Jahre bis zur Gründung des Forschungsverbunds Sonnenenergie - heute Forschungsverbund Erneuerbare Energien (FVEE) - durch das BMBF Anfang der 1990er Jahre. Der zweite Teil betrachtet die Zeit von 1990 bis 2015, wobei sich das Projekt auch hier auf das Spannungsfeld zwischen den wissenschaftlichen Entwicklungen und den politischen Aktivitäten fokussiert. Die Zeitabschnitte als Übersicht: Teil I:

I 1970 bis 1981 (bis zur Gründung des Fraunhofer ISE in Freiburg)

I 1981 bis 1986 (bis zur Reaktorkatastrophe in Tschernobyl)

I 1986 bis 1990 (Gründung des Forschungsverbunds Sonnenenergie)

Teil II:

I 1990 bis 1998 (bis zum Beginn der Regierungsbeteiligung von Bündnis90/Die Grünen)

I 1999 bis 2011 (bis zur Reaktorkatastrophe in Fukushima)

I 2011 bis 2015 (bis zur Weltklimakonferenz 2015 in Paris)

\section{Thesen und Leitfragen}

- Warum konnte sich vor allem in Deutschland eine weltweit anerkannte Spitzenforschung für Nutzungstechnologien erneuerbarer Energiequellen entwickeln?

- Umwelt- und Energieprobleme haben in den 1960er und 1970er Jahren zu einem Bewusstseins- und einem ökologi-

\section{„Warum konnte sich vor allem in Deutschland eine weltweit anerkannte Spitzenforschung für Nutzungstechnologien erneuerbarer Energiequellen entwickeln?"}

schen Wertewandel in der Bundesrepublik geführt. Das führte bei vielen Wissenschaftler/innen zu einem Umdenken.

I Die Energiepolitik in der Bundesrepublik wurde durch politische Bewegungen, Umweltorganisationen und Ressourcenprobleme getrieben. Wissenschaftler/innen haben darauf reagiert. An Orten, in denen Atomkraftgegner/innen besonders konstruktiv agierten, entstanden Forschungsinstitutionen für solare Energien.

I Großforschungseinrichtungen wie das DFVLR in Stuttgart (heute DLR - Deutsches Zentrum für Luft- und Raumfahrt) und die Kernforschungsanlage Jülich (KFA), eigentlich für Raumfahrt und Kernkraft eingerichtet, bildeten aufgrund ihrer Energieforschungskompetenzen institutionelle Keimzellen der Forschung und Entwicklung der erneuerbaren Energien. Sie erwiesen sich als wichtige Partner für das Forschungsministerium, um Forschungs- und Entwicklungsprojekte zusammen mit universitären Gruppen bearbeiten zu können.

I Welche Rolle spielte das damalige Bundesministerium für Forschung und Technologie (BMFT) in den 1970er Jahren?

I Die Folgen der weltweiten Ölkrisen waren ambivalent: Zum einen setzte die Suche nach alternativen Energiequellen sowie Möglichkeiten der Energieeinsparung ein und zum anderen die Suche nach einem Wirtschaftssystem, das sich weitgehend ohne staatliche und gewerkschaftliche Regulierungseingriffe - allein auf den Markt konzentrieren sollte: Es war der Beginn des sogenannten neoliberalen Wirtschaftssystems. Die ersten Energieforschungsprogramme des BMFT fielen in diese technologie- und wirtschaftspolitische Umbruchszeit. Das vertraute lineare Denksystem der Energieversorgung war zwar zusammengebrochen aber das neoliberale Wirtschaftssystem war noch nicht angekommen. In diesem wirtschafts- und energiepolitischen „Vakuum “ entstanden Spielräume, die eine Entwicklung in Richtung Energieeffizienz und erneuerbare Energien ermöglichten.

I Welche neuen naturwissenschaftlichen Erkenntnisse sowie ökonomischen und geisteswissenschaftlichen Überlegungen ermöglichten die rasche Entwicklung der Erneuerbaren und machten eine weitere Erforschung und Entwicklung attraktiv und erfolgversprechend? 
- Die Solarenergieforscher/innen haben mit der Entwicklung der Technologien zur Nutzung erneuerbarer Energiequellen neue Handlungsoptionen geöffnet und damit die Argumentation des Sachzwangs - wie zum Beispiel „wir haben eine Energielücke, also brauchen wir Kernkraftwerke“ - zerstört. Wurde die Gefahr einer Entwicklung der Demokratie zur Technokratie dadurch gemindert? Auf jeden Fall wurde der Spielraum für demokratische Entscheidung erweitert, beziehungsweise erst wieder ermöglicht.

- Die Reaktorkatastrophe von Tschernobyl führte zu einer energiepolitischen Zeitenwende in Westdeutschland. Verstärkt wurde diese Wende durch die drei Jahre später einsetzende revolutionäre Wende in Osteuropa. Beide Ereignisse, die nach Joachim Radkau (2011) zusammengehören, führten aufgrund gravierender Umweltprobleme in Ost und West (insbesondere in der ehemaligen DDR) zu einem beschleunigten Ausbau der erneuerbaren Energien, zur Gründung neuer Energieforschungsinstitute und zur Einrichtung des Forschungsverbunds Sonnenenergie (FVS) im Jahre 1990.

- Der Klimawandel als Argumentationslinie für die Notwendigkeit einer Energiewende erhielt erst nach dem Erdgipfel in Rio von 1992 größere Bedeutung. Er löste den Blick auf die Endlichkeit der Ressourcen ab.

- Wäre die demokratische Entwicklung in der Bundesrepublik ohne erneuerbare Energien ins Stocken geraten? Haben die erneuerbaren Energien, der Wertewandel und die ökologische Bewegung die Bundesrepublik demokratisiert und wurde damit ein Beitrag geleistet zur Entspannung des kalten Krieges? Hätte sonst der „Atomstaat“ (Robert Jungk) obsiegt oder hätte der „Radioaktive Zerfall der Grundrechte“ (Alexander Roßnagel) zu einem bürokratischen Absolutismus geführt?

- Wissenschaft ist für eine Demokratie lebenswichtig. Einerseits bieten Demokratien den Wissenschaften die Freiheiten, die sie benötigen, um frei forschen und lehren $\mathrm{zu}$ können; umgekehrt tragen die Wissenschaften zur Weiterentwicklung der Demokratie bei. Der amerikanische Klimaforscher Jonathan Foley hat eine ungewöhnlich scharfe Warnung an Trump gerichtet: „Do not mess with us“, schreibt er, „the War on Facts is a War on Democracy“. Anhand der Energieforschung und der Entwicklung solarer und erneuerbarer Energien soll gezeigt werden, dass eine Demokratie Wissenschaft und Forschung zwingend benötigt, um stabil zu bleiben.

- Was können wir lernen? Kann die Energieforschung für erneuerbare Energien als Transformationsmodell für mehr Nachhaltigkeit auch in anderen Wissenschaftsbereichen dienen?

\section{Politische und kulturelle Hintergründe}

Welche Rolle spielte die Forschungsförderpolitik in den verschiedenen Jahrzehnten? Wie verhielten sich die Energie- beziehungsweise Solarwirtschaft und wie die Energieversorger?
Welche Gegenkräfte haben sich formiert und wie haben sie sich bis heute entwickelt?

Aus den Interviews und aus der Literatur lassen sich Antworten auf diese Fragen herauslesen. Besonders interessant scheint der Impuls zu sein, der von der Studierendenbewegung der 1960 und 1970er Jahre ausging. Verschiedene Wissenschaftler/innen berichten, dass sie diese Bewegung politisiert habe. Denn vorgegebene Denkrichtungen wurden nicht mehr einfach hingenommen, es wurde bewusst nach Alternativen zur Kernenergie und Kernfusion gesucht. Es war die Zeit, in der der ökologische Wertewandel in Gang kam und man sich verschiedener Umweltsünden bewusst wurde. Schon im Wahlkampf der SPD gegen die CDU mit Kanzler Adenauer im Jahre 1961 sagte Willy Brandt in Bonn: „Der Himmel über der Ruhr muss wieder blau werden“.

Es war sowohl die Zeit der Notstandsgesetze als auch eine Zeit des Aufbruchs, „mehr Demokratie zu wagen“, wie Willy Brandt 1969 in seiner Regierungserklärung sagte. Es war die Zeit gesellschaftlicher Experimente: Wohngemeinschaften, Tabubrüche, Experimente in Wissenschaft und Kultur, in Musik und Kunst wie zum Beispiel die Theaterexperimente von Peter Stein an der Berliner Schaubühne, das Antitheater mit Rainer Werner Fassbinder und seine berühmten Filme, Alexander Kluge, Joseph Beuys und Klaus Staeck mit der Selbstorganisation der Künstler/innen und für eine Kunst für Alle. Kurz, es war der Beginn einer Bewegung, die alles infrage stellte.

\section{Die Rolle der Forschungsförderung}

Auch die Politik hat reagiert. Forschungsminister Horst Ehmke gründete als erster Minister des neu gegründeten Bundesministeriums für Forschung und Technologie (BMFT) ein Referat für nichtnukleare, nichtfossile Energien.

Im neuen Referat „Erneuerbare Energien und Rationale Energieverwendung“ ging es aber zunächst nur darum, die Abhängigkeit von Kohle und Öl zu reduzieren, Kohletechnologien umweltfreundlicher zu gestalten und da, wo es ökonomisch und ökologisch möglich erschien, auch erneuerbare Energien einzusetzen. Es ging damals also keineswegs um die Ablösung der Kernenergie, sondern darum, aus Ressourcenund Umweltgründen Erdöl und Erdgas aus der Versorgung soweit wie möglich herauszunehmen. Schwerpunkt der BMFTForschungsförderung war die rationelle Energieverwendung, um den Bedarf zunächst mit konventionellen Techniken zu reduzieren. Der restliche Bedarf sollte unter Schonung von Umwelt und Ressourcen auch mit Abwärme, Sonnen- und Windenergie gedeckt werden.

Es ist aufschlussreich, dass 1975, also bereits ein Jahr später, für das BMFT eine erste Programmstudie über die Energiequellen von morgen von einem Konsortium aus DFVLR (heute DLR) und KFA Jülich und verschiedenen Industriefirmen erstellt wurde. Seit dieser Zeit gibt es in Deutschland eine kontinuierliche, wenn auch mit vielen Auf- und Abwärtsbewegungen - je nach Ölpreis - Förderung der erneuerbaren Energien, 
die damals noch nicht so hießen, sondern als „nichtnukleare und nichtfossile Energien" bezeichnet wurden. 1980 wurde mit dem Fraunhofer ISE das erste außeruniversitäre Forschungsinstitut für Solare Energiesysteme gegründet, dass sich ausschließlich solaren Themen widmete.

Die seit dem Energieforschungsprogramm 1974 stetige Forschungsförderung - zunächst weitgehend auf Energieeinsparung und Effizienz beschränkt - erklärt zu einem gewissen Grade das hohe Niveau der Energietechnologien in Deutschland. Doch die Wissenschaftler/innen mussten viel Unverständnis und Spott ertragen: „Wie wollen die mit diesen geringen Energiedichten aus Sonne und Wind die Energieversorgung einer Industriegesellschaft gewährleisten?", hieß es. Und es blieb nicht beim Spott, es kam auch zu Anfeindungen. Viele Wissenschaftler/innen und Professor/innen mussten um ihre Reputation bangen. Doch sie blieben standhaft. Ihre wissenschaftlichen Überzeugungen waren ihnen wichtiger als die soziale Anerkennung. Diese Wissenschaftler/innen arbeiteten nicht mehr in einem Elfenbeinturm, sondern sie haben energiepolitische Positionen bezogen. Sie haben Verantwortung für kommende Generationen übernommen und waren, ob sie es wollten oder nicht, in der Wissenschaftspolitik angekommen.

\section{Die energiepolitische Zeitenwende}

1986 kam es zur energiepolitischen Zeitenwende: Die Reaktorkatastrophe in Tschernobyl veränderte alles - nichts war mehr wie vorher, auch wenn Reaktionen oft viele Jahre später erfolgten. Das Bundesumweltministerium aber wurde sofort gegründet und die Forschungsförderung für erneuerbare Energien wurde von heute auf morgen verdoppelt.

In der folgenden Zeit kam es zu einem Prozess der Institutionalisierung von Forschung und Entwicklung erneuerbarer Energie. Die meisten noch heute aktiven außeruniversitären Forschungseinrichtungen wurden gegründet, 1990 wurde auf Initiative des BMFT der Forschungsverbund Sonnenenergie (FVS) gebildet und 1991 trat das erste Stromeinspeisegesetz in Kraft.

Aber erst mit der Regierungsbeteiligung der Grünen/Bündnis90 ab 1998 wurde der entscheidende energiepolitische Umbruch eingeleitet, der seit Tschernobyl auf sich warten ließ: Ein Vertrag zum Atomausstieg wurde geschlossen und der Bundestag verabschiedete das bundesweite Gesetz zur kostendeckenden Einspeisevergütung für erneuerbaren Strom, das EEG. Mit diesen politischen Maßnahmen wurde das Fundament gelegt für den energiewirtschaftlichen Umbau einer Industriegesellschaft, um sie auf neue, auf nachhaltige Füße zu stellen. Das EEG übernahm aber auch die Funktion einer ForschungsTechnologieförderung, sodass es zu einer raschen Technologieentwicklung zur Nutzung der erneuerbaren Energiequellen kam.

Die energiepolitische Zeitenwende, die 1986 begann, hat sich mit den drei Reaktorkatastrophen in Fukushima 2011 ein weiteres Mal beschleunigt. Der Atomausstieg aus dem Jahre

\section{„Ganz wesentlich ist also eine gegenseitige Kommunikation zwischen Wissenschaft und Gesellschaft, und zwar in beide Richtungen."}

2000 wurde 2011 parlamentarisch besiegelt und ein Konzept für die Energiewende von der CDU/SPD-Regierung verabschiedet. Aber nun wurden auch die Zeichen des Klimawandels sichtbarer und fühlbarer. Mit der Verabschiedung des Weltklimavertrags der Vereinten Nationen in Paris am 12. Dezember 2015 durch 195 Staaten ist die weltweite Energiewende nicht mehr rückgängig zu machen. Dies liegt nicht zuletzt daran, dass die Erneuerbaren dank Forschung und Technologieentwicklung vor allem auch durch die deutsche Wissenschaft gesellschaftlich und ökonomisch attraktiver geworden sind als die fossilen und nuklearen Energien.

\section{Wechselwirkung von Forschung und Gesellschaft}

Die Forschung und Entwicklung der Erneuerbaren zeigt, dass die Wissensproduktion heute nicht mehr in einem „Elfenbeinturm“ erfolgen kann, sondern dass sie nur in politischen, ökonomischen, ökologischen und sozialen Zusammenhängen gesellschaftlich effizient und auf Dauer finanzierbar ist.

Die sozialen Argumente haben es dabei immer am schwersten, ernst genommen zu werden. Doch mit Technik allein können wir Energieprobleme nicht lösen. Es geht ja nicht nur um die Technikentwicklung für erneuerbare Energietechniken, sondern auch darum wahrzunehmen, wer diese Techniken benutzen soll, wie sie eingesetzt werden können, was sie kosten dürfen und wie umweltfreundlich sie sind. Der Einfluss der Nutzer/innen, der Anwender/innen und der Erfahrungen sorgt für eine social shaped technology, wie es in der Europäischen Union (EU) heißt.

Den Wissenschaftler/innen geht es darum, diesen gesellschaftlichen Prozess mitgestalten. Sie sind keine dienstbaren Zwerge, die alles erforschen, was gewollt wird, sondern in einer freiheitlichen Demokratie wollen sie mitreden. Dabei handelt es sich um eine strategische Wechselwirkung, an der nicht nur die Forscher/innen und Entwickler/innen, sondern sämtliche mit ihnen verbundenen Forschungseinrichtungen teilnehmen: - Gesellschaft: Umweltverbände, Kirchen, Gewerkschaften etc. I Politik: Parlamente, Ministerien, Nichtregierungsorganisationen 
- Industrie und Wirtschaft

I Kapitalwirtschaft: Kreditinstitute, Risikokapitalgeber etc.

- Forschung: Universitäten, private Forschung etc.

Umweltverbände betreiben das Agenda Setting, Kirchen predigen die Erhaltung der Schöpfung, Gewerkschaften gestalten die Arbeitsbedingungen der Wissenschaftler/innen und Techniker/innen mit. Diese gesellschaftlichen Kräfte haben eine große Bedeutung. Die Arbeitsbedingungen haben Einfluss darauf, ob und welche Mitarbeiter/innen gewonnen werden können. Dies wirkt sich wiederum auf die Qualität der Forschung aus. Die Forschungsförderung entscheidet, welche Finanzvolumina der Energieforschung und ihren einzelnen Sparten (konventionelle, regenerative Energien) zur Verfügung stehen.

Ganz wesentlich ist also eine gegenseitige Kommunikation zwischen Wissenschaft und Gesellschaft, und zwar in beide Richtungen. Der 1990 gegründete Forschungsverbund Sonnenenergie (FVS) steht an der Schnittstelle zwischen Gesellschaft und Wissenschaft und nimmt die Funktion einer kommunikativen Brücke zwischen Politik und Wissenschaft wahr.

\section{Erneuerbare Energien und Demokratisierung der Gesellschaft}

Es ist interessant, sich zu vergegenwärtigen, in welchen Ländern sich die solaren und regenerativen Energien entwickelten: Es waren demokratische Länder, in denen die zivilgesellschaftlichen und wissenschaftlichen Freiheiten breiten Raum boten für neue Ideen, kreative, alternative Techniken. Und es waren die Länder, in denen die Studentenbewegung der 1960er Jahre zu Demokratisierungsprozessen in den Universitäten führten und im wahrsten Sinne des Wortes Spielräume eröffneten, nicht nur für soziale, sondern auch für technologische Innovationen.

Letztlich führten sie auch zu neuen politischen Parteien und Regierungskonstellationen in der BRD. Der Kampf für die erneuerbaren Energien, für die Möglichkeit einer Energiewende war immer auch ein Kampf gegen technologische Sachzwänge und industriepolitische Einseitigkeit. Ihm ist es zu verdanken, dass es heute eine freie Wahl der Energieversorgung gibt.

In der DDR, um einen Vergleich zu einem autoritären Staat zu ziehen, tat sich in Richtung erneuerbarer Energien grundsätzlich nichts. Zwar gab es einzelne Initiativen, Photovoltaik und Solarthermie zu nutzen, aber Forschung und Entwicklung wurden nicht betrieben und nicht gefördert. In der Bundesrepublik dagegen haben die Solarenergieforscher/innen mit der Entwicklung der Technologien zur Nutzung erneuerbarer Energiequellen neue Handlungsoptionen geöffnet und damit die Sachzwangsargumentation zerstört. Die Gefahr einer Entwicklung der Demokratie zur Technokratie wurde gemindert. Damit wurde ein Spielraum für demokratische Entscheidung erweitert beziehungsweise geöffnet.

Die gewaltlose Revolution in der DDR 1989 traf auf eine evolutionär demokratisierte und reformierte Bundesrepublik. Eine in ihrem Wesen sozialdemokratische Bundesrepublik wurde gesellschaftliches Vorbild für viele DDR-Bürger/innen, sodass das von der SED propagierte Feindbild einer profaschistischen und in seiner Wirklichkeit zutiefst undemokratischen kapitalistischen Gesellschaft in Westdeutschland nicht mehr verfing.

Kann die Frage gestellt werden, ob wir in Deutschland ohne Wissenschaft und Forschung der erneuerbaren Energien sowie ohne die Entwicklung des Produktionsstandorts für Photovoltaik, Bioenergie und Windenergie heute keine Zivilgesellschaft mehr hätten, beziehungsweise es heute keine demokratische Gesellschaft mehr in Deutschland gäbe?

Diese Frage ist sehr zugespitzt formuliert. Eine demokratische Gesellschaft hängt bestimmt nicht nur von der Entwicklung der Erneuerbaren ab. Aber der Blick auf den Kampf gegen die Atomenergie zeigt, dass sich die Antiatomkraftbewegung $\mathrm{zu}$ einer Energiebewegung für erneuerbare Energien entwickelte und damit zu einer Demokratisierung der Energiegewinnung und -versorgung geführt hat. Somit wurde Demokratie nicht nur erhalten, sondern hat sich auch weiterentwickelt. Die Bücher von Robert Jungk „Der Atomstaat - Fortschritt in die Unmenschlichkeit“ und „Radioaktiver Zerfall der Grundrechte“ von Alexander Roßnagel zeigen, wohin sich die Dinge hätten entwickeln können. Haben also die erneuerbaren Energien, der ökologische Wertewandel und die Umweltbewegung die Bundesrepublik demokratisiert und wurde damit ein Stück kalten Krieges abgebaut?

Von der Öffentlichkeit wenig beachtet hat die Enquetekommission „Die Zukunft der Kernenergie“ des Bundestages von 1979 bis 1982 das Kriterium der Sozialverträglichkeit von Energiesystemen akzeptiert und eine grundsätzliche Weiche gestellt, die weg von der Technologie der Schnellen Brüter führte und damit den Weg zu Optionen der Energieeinsparung und der erneuerbaren Energien grundsätzlich öffnete.

Doch warum fanden diese Entwicklungen vor allem in Deutschland statt? Warum gab es hier die längste Antiatomkraftbewegung aller Industriestaaten auf der Welt? Was waren die politischen Hintergründe? Prof. Joachim Radkau hat in seinem Buch „Die Ära der Ökologie“ versucht, darauf Antworten zu geben. Aber er hat wohl noch nicht alle Antworten gefunden, wichtige fehlen noch.

\section{Literatur \\ Radkau, J. (2011): Die Ära der Ökologie. Bonn.}

\section{AUTOR + KONTAKT}

Dr. Gerd Stadermann ist Fellow am Institut für ökologische Wirtschaftsforschung (IÖW) und Wissenschaftlicher Beirat in der Deutschen Gesellschaft für Sonnenenergie (DCS).

Dr. Gerd Stadermann, E-Mail: post@wissenschaft-politik-stadermann.de

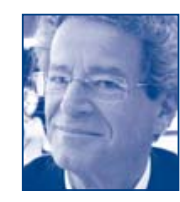

